\title{
Determination of Partial Photochemical Quantum Yields of Reversible Photoisomerizations of Stilbene-1 Derivatives
}

\author{
G. Gauglitz, R. Goes, W. Stooß, and R. Raue* \\ Institut für Physikalische und Theoretische Chemie, Universität Tübingen
}

Z. Naturforsch. 40 a, 317-323 (1985); received December 10, 1984

Dedicated to Prof. Schulte-Frohlinde on the occasion of his 60th anniversary

\begin{abstract}
Partial photochemical quantum yields of derivatives of stilbene-1 can be determined by a new microprocessor controlled apparatus for the measurement of fluorescence intensities in dependence on reaction time. The consecutive photoproducts must not be known. Some of the 20 examined dyes show laser activity combined with relatively high photostability. Photochemical quantum yields and laser peak power were determined in dependence on structure. A detailed knowledge of the photokinetic parameters gives the opportunity to obtain indications of efficient and photostable laser dyes.
\end{abstract}

\section{Introduction}

The quality of laser dyes depends on their photophysical and photochemical properties. Whereas the photophysical features are well known, the photochemical degradation steps are quite unknown. In the case of coumarine derivatives detailed examinations have been made [1]. Even though the photobleaching rates of a number of dyes have been determined [2-4], these results can not reveal photochemical characteristics such as the rate determining step. Therefore the partial photochemical quantum yields [5] of all the reaction steps have to be determined. In the case of stilbene- 1 type laser dyes it has recently been shown [6] that a reversible photoisomerization is the photodegradation step which controls the laser activity. Its dependence on the type of substitution of the stilbene-1 frame was found by calculation of the partial photochemical quantum yield of this step. If absorbance measurements are used for the kinetic analysis, these calculations also require the knowledge of the first consecutive photoproduct. In the past, these values have been obtained either by measurements at different photostationary states [7], trial and error methods in combination with three photostationary states [8] or by iterative procedures

\footnotetext{
* Bayer AG, D-5090 Leverkusen.
}

Reprint requests to Prof. Dr. G. Gauglitz, Institut für Physikalische Chemie der Universität Tübingen, Auf der Morgenstelle 8, 7400 Tübingen.
[9]. In all cases constancy of the photostationary state was a prerequisite. This approximation was not valid in our case since all the derivatives showed a rather fast additional photoreaction.

\section{Theory}

The photochemical pathway for the derivatives of stilbene-1 is a consecutive multi-step process [5], where the first step is a reversible trans $\leftrightarrow$ cis photoisomerization [6]. Intensity diagrams $[10,11]$ show that the trans isomer is the only fluorescent reactant in the whole reaction path. Therefore the following mechanism can be assumed (superscript $F$ means "fluorescent"):

$$
\operatorname{trans}\left(\mathrm{A}^{\mathrm{F}}\right) \frac{\varphi_{1}^{\mathrm{A}}}{\varphi_{2}^{\mathrm{B}}} \operatorname{cis}(\mathrm{B}) \stackrel{\varphi_{3}^{\mathrm{B}}}{\rightarrow} \text { product }(\mathrm{C}) \rightarrow \rightarrow \text {. }
$$

An evaluation of the absorbance diagrams indicates that the rates $\varphi_{3}^{\mathrm{B}}$ etc. of the consecutive photoreactions are smaller than $\varphi_{1}^{\mathrm{A}}$ and $\varphi_{2}^{\mathrm{B}}$. Since the partial photochemical quantum yield $\varphi_{1}^{\mathrm{A}}$ for the photodegradation of $\mathrm{A}$ was of main interest, only the two linearly dependent steps $\mathrm{A}^{\mathrm{F}} \rightarrow \mathrm{B}$ and $\mathrm{B} \rightarrow \mathrm{A}^{\mathrm{F}}$ of the first linearly independent step $\mathrm{A}^{\mathrm{F}} \rightleftharpoons \mathrm{B}$ were taken into account in the rate law

$$
\dot{a}(t)=-I F\left\{R_{1} \cdot a(t)-R_{2}[a(0)-a(t)]\right\}
$$

with

$$
R_{1}=\varepsilon_{\mathrm{A}}^{\prime} \cdot \varphi_{1}^{\mathrm{A}}, \quad R_{2}=\varepsilon_{\mathrm{B}}^{\prime} \cdot \varphi_{2}^{\mathrm{B}},
$$


where $\varepsilon_{A}^{\prime}$ and $\varepsilon_{B}^{\prime}$ are the absorptivities of trans and cis isomer, $F=\left(1-10^{-E^{\prime}}\right) / E^{\prime}$ is the reciprocal value of the photokinetic factor, and $I=2.303 \cdot 10^{3} \cdot I_{0}$ (Einstein $\mathrm{cm} \mathrm{l}^{-1} \mathrm{~s}^{-1}$ ) depends on the intensity of the light source. The factor 2.303 takes into account that in the formula for the absorbed light an exponential function occurs. It converts this natural to decadic absorptivities [10]. In case of an absorbance measurement only the sum $\left(R_{1}+R_{2}\right)$ is obtained by a kinetic analysis. If the absorptivity $\varepsilon_{\mathrm{B}}^{\prime}$ of the first photoproduct (cis-isomer) is unknown, neither $\varphi_{1}^{\mathrm{A}}$ nor $\varphi_{2}^{\mathrm{B}}$ can be evaluated. But the measurement of the fluorescence intensity $I_{\alpha}^{\mathrm{F}}(t)$ at an observation wavelength $\alpha$ can be used to calculate the first partial quantum yield directly. The absorbance $E^{\prime}$ at irradiation (and excitation) wavelength for very dilute solutions becomes very small $(\ll 1)$ and $F$ approaches 1 . Therefore the fluorescence depends on the trans concentration only [10]. With

$$
F \approx 1 \text { and } I_{\alpha}^{\mathrm{F}}(t) \sim a(t)
$$

the rate law is given by

$$
\dot{I}_{\alpha}^{\mathrm{F}}(t)=-\left(R_{1}+R_{2}\right) I I_{\alpha}^{\mathrm{F}}(t)+R_{2} I I_{\alpha}^{\mathrm{F}}(0) .
$$

In the photostationary state $(s), I_{\alpha}^{\mathrm{F}}(s)$ becomes zero and therefore

$$
I_{\alpha}^{\mathrm{F}}(s)=\frac{R_{2}}{R_{1}+R_{2}} I_{\alpha}^{\mathrm{F}}(0) .
$$

From (3) and (4) follows the differential equation

$$
I_{\alpha}^{\mathrm{F}}(t)=-I\left(R_{1}+R_{2}\right)\left[I_{\alpha}^{\mathrm{F}}(t)-I_{\alpha}^{\mathrm{F}}(s)\right],
$$

which can be integrated to yield

$$
\begin{aligned}
\ln \left[I_{\alpha}^{\mathrm{F}}(t)-I_{\alpha}^{\mathrm{F}}(s)\right]= & \ln \left[I_{\alpha}^{\mathrm{F}}(0)-I_{\alpha}^{\mathrm{F}}(s)\right] \\
& -I\left(R_{1}+R_{2}\right) t .
\end{aligned}
$$

The slope of a diagram $\ln \left[I_{\alpha}^{\mathrm{F}}(t)-I_{\alpha}^{\mathrm{F}}(s)\right]$ vs. $t$ is given by

$$
-m=\left(R_{1}+R_{2}\right) \cdot I=I R_{2} \frac{I_{\alpha}^{\mathrm{F}}(0)}{I_{\alpha}^{\mathrm{F}}(s)} .
$$

This slope can be used to calculate the partial photochemical quantum yields

$$
\varphi_{2}^{\mathrm{B}}=\frac{-m}{I \varepsilon_{\mathrm{B}}^{\prime}} \frac{I_{\alpha}^{\mathrm{F}}(s)}{I_{\alpha}^{\mathrm{F}}(0)}
$$

and, from (4) and (7),

$$
\varphi_{1}^{\mathrm{A}}=\frac{-m}{I \varepsilon_{\mathrm{A}}^{\prime}} \frac{I_{\alpha}^{\mathrm{F}}(0)-I_{\alpha}^{\mathrm{F}}(s)}{I_{\alpha}^{\mathrm{F}}(0)} .
$$

Whereas $\varphi_{1}^{\mathrm{A}}$ can be calculated directly, the correlated partial photochemical quantum yield $\varphi_{2}^{\mathrm{B}}$ has to be estimated by combined absorbance $\left(\lambda^{\prime}\right)$ and fluorescence $(\alpha)$ measurements by use of the formulae

$$
\begin{aligned}
& b(s)=a(0)-a(s), \quad E(s)=\varepsilon_{\mathrm{A}}^{\prime} a(s)+\varepsilon_{\mathrm{B}}^{\prime} b(s), \\
& a(s) / a(0)=I_{\alpha}^{\mathrm{F}}(s) / I_{\alpha}^{\mathrm{F}}(0) .
\end{aligned}
$$

Furthermore, the percentage of cis-isomer at the photostationary state is given by

$$
\% \operatorname{cis}(s)=\frac{I_{\alpha}^{\mathrm{F}}(0)-I_{\alpha}^{\mathrm{F}}(s)}{I_{\alpha}^{\mathrm{F}}(0)} \cdot 100
$$

and a degradation rate $\left(\mathrm{s}^{-1}\right)$ by

$$
U=\frac{I_{\alpha}^{\mathrm{F}}(0)-I_{\alpha}^{\mathrm{F}}(s)}{I_{\alpha}^{\mathrm{F}}(0)} \frac{I_{\mathrm{r}} E_{\mathrm{r}}}{I E(0) \Delta t},
$$

which is comparable amongst different dyes, because a relative intensity of $2.303 \cdot 10^{-6}$ (Einstein $\mathrm{cm} \mathrm{l}^{-1} \mathrm{~s}^{-1}$ ) and a relative absorbance $E_{\mathrm{r}}=0.02$ are used to obtain relative rates.

\section{Experimental}

The fluorescence intensities were measured with respect to irradiation time by a ZFM4/PMQ II setup (Carl Zeiss, Oberkochen) in a self-constructed cell compartment (Figure 1). The stabilized light source St 46 (mercury-arc, Quarzlampengesellschaft Hanau) was used both for photo-irradiation and excitation of fluorescence. Its intensity is controlled by a photodiode via a beamsplitter. The fluorescence spectra are dispersed by a monochromator M4Q (Carl Zeiss). The excitation and irradiation wavelength $\lambda^{\prime}$ is selected by an UV-PIL interference filter (Schott \& Gen, Mainz). The absorbance of the stirred sample at this wavelength $E^{\prime}$ can be monitored via the optical pathway $A$ in comparison to the blank $V$ (solvent). The whole set-up is standardized by a luminescent block of uranyl glass at $\alpha=535 \mathrm{~nm}$ to a read-out of the amplifier of $100 \%$. Therefore the single beam method becomes reproducible with respect to amplification and excitation intensity. The analog signal of the amplifier is recorded by a chart recorder and digitized by an A/D-converter, which is part of a Lab Data Station [12]. This is also used for the kinetic evaluation and the graphics. The intensity $I_{0}$ of the light source is determined absolutely by standard chemical actinometry $[13,14]$. 


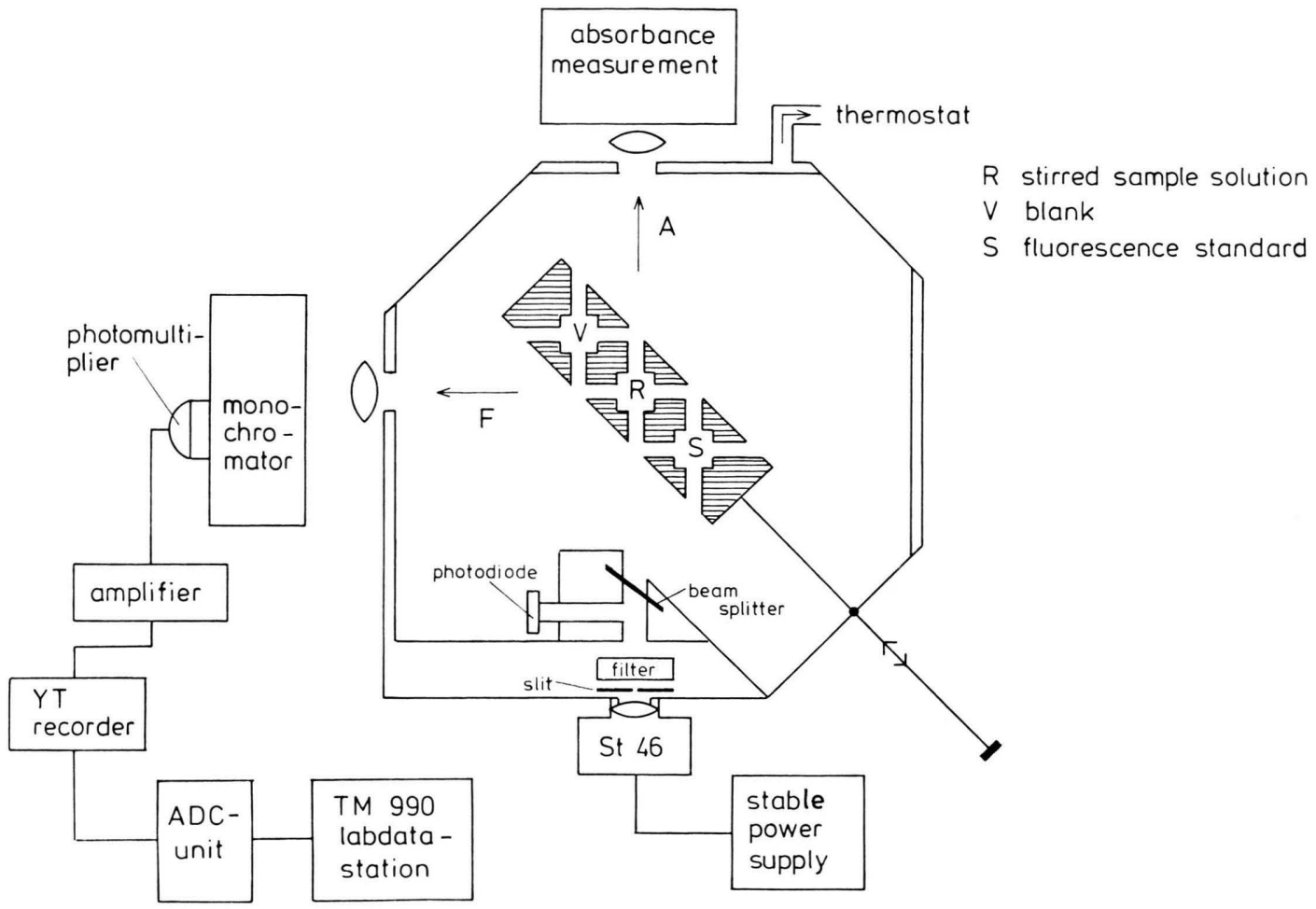

Fig. 1. Cell for combined measurement of the fluorescence intensity (F) and the absorbance (A) of the sample (R), an uranyl glass standard (S) and the solvent (V).

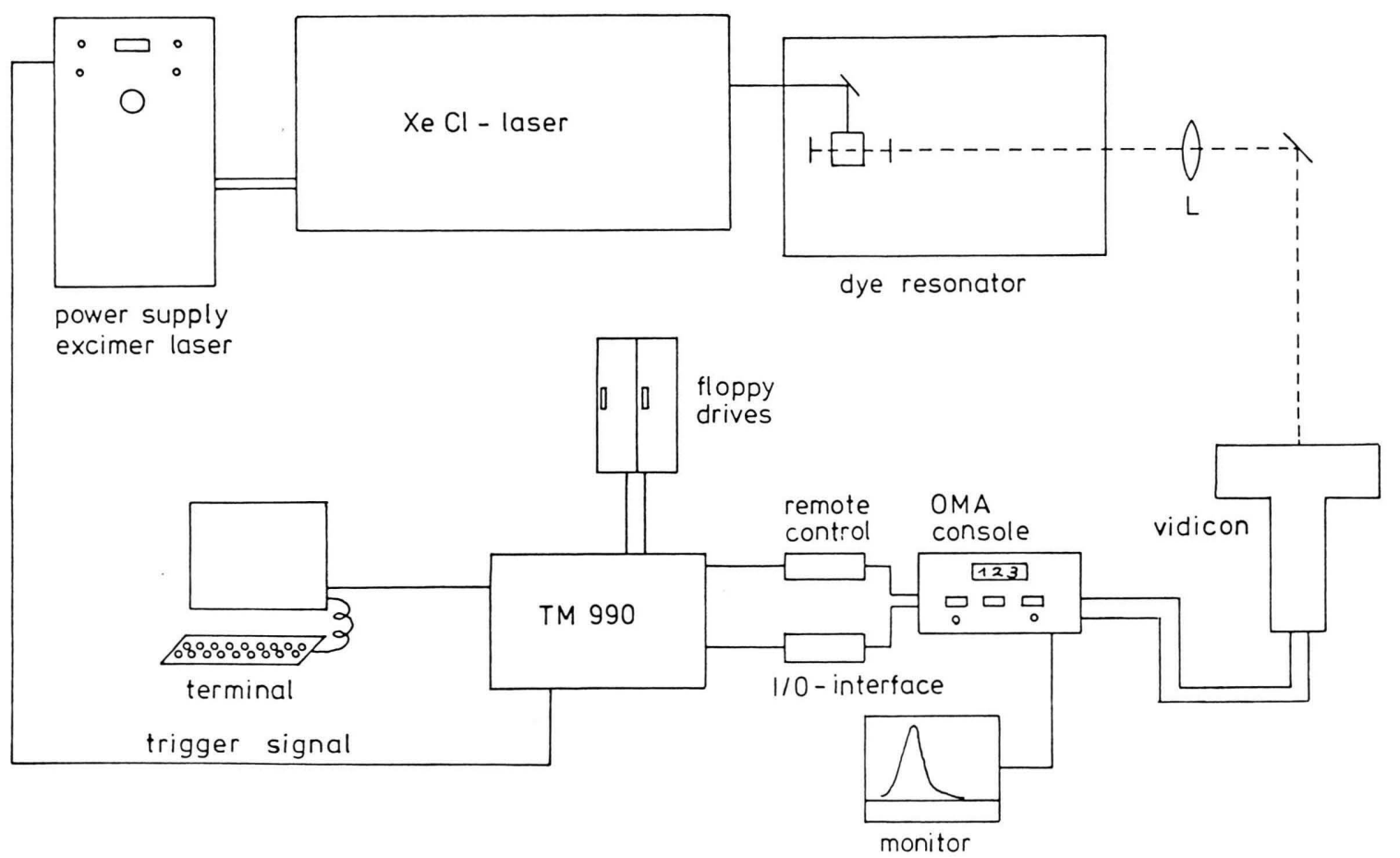

Fig. 2. Block diagram of the laser set-up. Excimer laser EMG 101 with FL 2000, optical multichannel analyzer (OMA) and process control unit (TI 990 microprocessor). 
The measurements of the laser efficiencies were realized by an excimer laser EMG 101 at $308 \mathrm{~nm}$ (60 mbar Xe, $1110 \mathrm{mbar} \mathrm{Ar}, 80 \mathrm{mbar} \mathrm{He} / \mathrm{HCl}$, Lambda Physik, Göttingen) and a dye resonator FL 2000. Peak power and tuning range of the dyes were determined by a bolometer (Laser Instrumentation model 142, Chertsey, England) and an OMAsystem [15] (EG\& G, Munich) respectively. A block diagram of the set-up is given in Figure 2.

\section{Results and Discussion}

In Table 1 the code name, the molecular weight and the structure of all the examined 20 stilbene- 1 derivatives and the stilbene- 1 frame are given. All dyes were synthesized in the dye laboratory of Bayer AG in Leverkusen. They are arranged in decreasing magnitude of laser efficiency. The measured relative values of the peak powers in ethylene glycol are summarized in comparison to Rhodamine $6 \mathrm{G}$ in Table 2.

The photokinetic data were obtained by measuring the change of fluorescence intensity in ethylene glycol during irradiation and calculating according to (7) and (8). The irradiation wavelength $\lambda^{\prime}=365 \mathrm{~nm}$ was chosen for two reasons. First the intensity of the light source at this wavelength was larger and caused a faster change in fluorescence. Secondly at this wavelength a larger amount of the cis isomer exists in the photostationary state. Both reasons increase the $\mathrm{S} / \mathrm{N}$-ratio and make the evaluation more accurate.

The partial photochemical quantum yields are independent of the irradiation wavelength between 313 and $365 \mathrm{~nm}$ as could be verified in different solvents for stilbene-1 [12]. Oxygen-free solutions show smaller photodegradation in the photoconsecutive step [16], but in practice aerated solutions have to be used. Therefore all experiments were done in air-saturated solutions. The observation wavelength $\alpha$ is selected at the maximum of the fluorescence spectrum. Two types of diagrams were evaluated. The first is a graph of $\ln I_{\alpha}^{\mathrm{F}}(t)$ vs. $t$, which shows two sections approximated by straight lines. The first slope corresponds to the first linearly independent step. The second corresponds to the reaction $B \rightarrow C$. Both slopes are used to estimate $I_{\alpha}^{\mathrm{F}}(s)$. In Fig. 3 this type of diagram is presented for some dyes to demonstrate the different behaviour.
Table 1. Code name, molecular weight and structure of the examined dyes in order of decreasing laser efficiency. Dyes in the lower part of the table (below the line) do not lase in ethylene glycol.

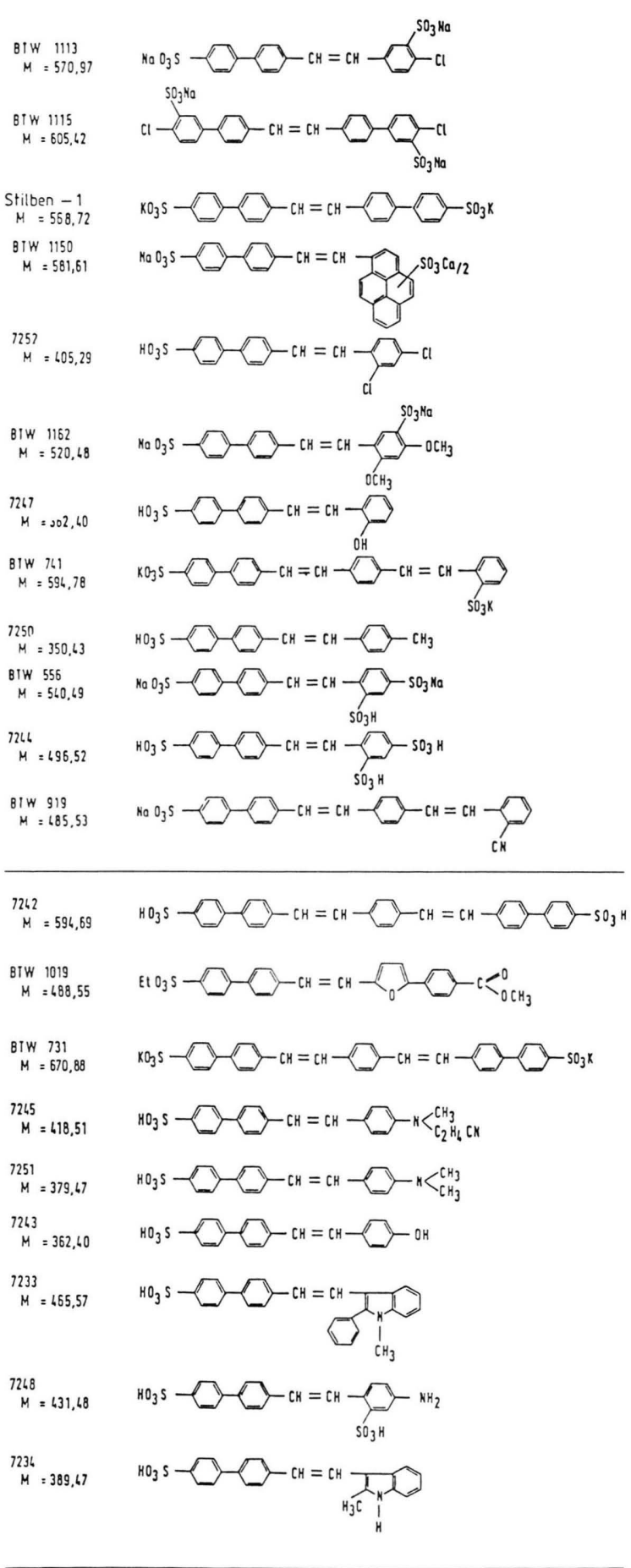




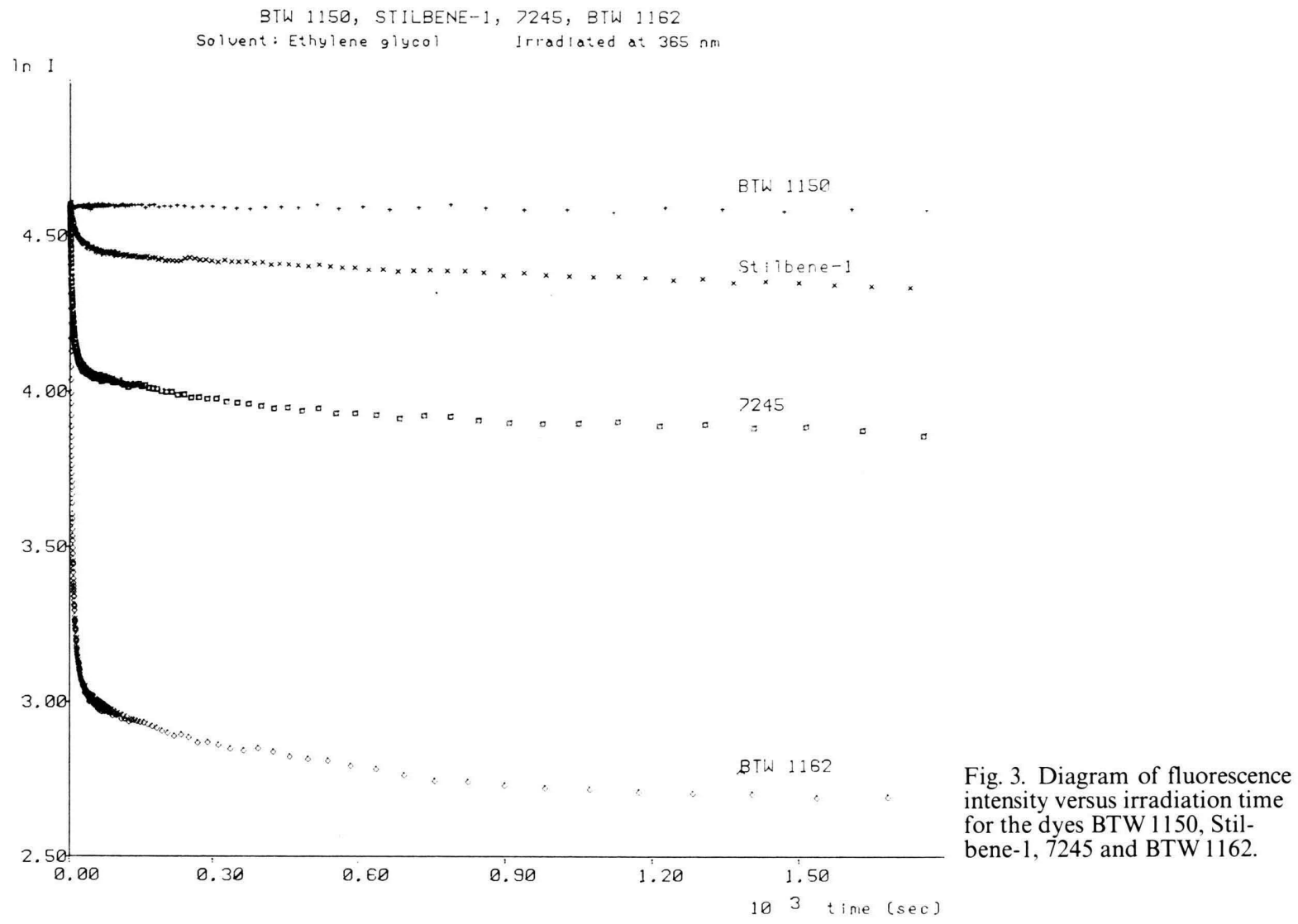

The second type of diagram is a plot of the natural logarithm of the difference between the fluorescence intensity at reaction time $t$ and that in the photostationary state $(s)$ vs. the irradiation time. The slope of this diagram $\left(\ln \left[I_{\alpha}^{\mathrm{F}}(t)-I_{\alpha}^{\mathrm{F}}(s)\right]\right.$ vs. $\left.t\right)$ correlates to the trans-cis photoreversible equilibrium of the first linearly independent step $\mathrm{A}^{\mathrm{F}} \rightleftharpoons \mathrm{B}$. From the first slope of such diagrams the partial photochemical quantum yields $\varphi_{1}^{\mathrm{A}}$ were determined according to (8). The "pseudo" [17] quantum yields $Q=R_{1}+R_{2}$ can be calculated from the slope of a diagram according to (6) for the first linearly independent step $\mathrm{A}^{\mathrm{F}} \rightleftharpoons \mathrm{B}$. The results are listed in Table 3 together with the percentage of cis-compound in the photostationary state (according to (9)) and the degradation rate $U$ of the trans isomer (10).

The "pseudo" quantum yield $Q$ depends on the irradiation wavelength (see (2)). Therefore $Q$ is not a useful parameter for detailed information. To get comparable values of $U$ the results were put in their relation to $E(0)$ and the intensity of irradiance according to (10). This parameter is quite easy to obtain, but is in principal only descriptive, since the amount of light absorbed is taken into consideration only indirectly. Both the rate of degradation and the amount of trans-component in the photostationary state depend to a great extent on the structure of the trans isomer. In many cases there is a correlation between the value of $\% \operatorname{cis}(s)$ and the values of $Q$ and $U$. Thus a high rate of disappearance of trans isomer causes a high percentage of cis-product in the equilibrium (e.g. BTW 1162, 7247). On the other hand, a low cis concentration must not mean a slow photochemical reaction (BTW 919, BTW 741). The behaviour of these derivatives can be explained by a rather high rate of the consecutive step from $B$ to $\mathrm{C}$, which is larger by one order of magnitude 
Table 2. Relative peak powers and $\alpha_{\max }$ of the lasing dyes in comparison to rhodamine $6 \mathrm{G}$, pumped by an excimer laser at $308 \mathrm{~nm}$ in ethylene glycol.

\begin{tabular}{lll}
\hline & $\begin{array}{l}\text { Rel. } \\
\text { peak power } \\
\%\end{array}$ & $\begin{array}{l}\alpha_{\max } \text { of } \\
\text { laser tuning } \\
\text { curve in [nm] }\end{array}$ \\
\hline $\begin{array}{l}\text { Rhodamine 6 G } \\
\text { BTW 1113 }\end{array}$ & 100.0 & 589 \\
BTW 1115 & 71.4 & 424 \\
Stilbene-1 & 63.6 & 420 \\
BTW 1150 & 53.5 & 416 \\
7252 & 35.6 & 474 \\
BTW 1162 & 16.4 & 400 \\
7247 & 15.0 & 445 \\
BTW 741 & 12.9 & 437 \\
7250 & 6.6 & 442 \\
BTW 556 & 4.0 & 420 \\
7244 & 3.8 & 425 \\
BTW 919 & 3.7 & 416 \\
\hline
\end{tabular}

Table 3. Photokinetic data of the stilbene-1 derivatives.

\begin{tabular}{lccccc}
\hline Code names & $\varphi_{1}^{\mathrm{A}} \cdot 10^{-2}$ & $Q \cdot 10^{3}$ & $\% \operatorname{cis}(s)$ & $U\left[\mathrm{~s}^{-1}\right]$ \\
\hline BTW 1150 & $<0.0001$ & $<0.1$ & - & - \\
BTW 1113 & $2.24 \pm 0.01$ & 5.77 & 17.0 & 0.75 \\
BTW 1115 & 2.59 & \pm 0.02 & 6.23 & 19.2 & 0.53 \\
Stilbene-1 & $1.57 \pm 0.02$ & 5.21 & 13.8 & 0.75 \\
7250 & $3.57 \pm 0.03$ & 2.19 & 16.6 & 0.60 \\
BTW 741 & $5.51 \pm 0.44$ & 6.38 & 4.4 & 1.74 \\
7247 & 17.7 & \pm 0.2 & 3.23 & 76.9 & 2.94 \\
BTW 919 & 19.8 & \pm 0.5 & 8.80 & 21.2 & 4.05 \\
7244 & 33.9 & \pm 0.6 & 8.38 & 58.6 & 5.90 \\
BTW 1162 & 38.9 & \pm 0.6 & 13.8 & 79.1 & 5.79 \\
7252 & 41.8 & \pm 0.3 & 5.94 & 85.8 & 6.84 \\
BTW 556 & 49.7 & \pm 0.4 & 10.4 & 80.3 & 5.27 \\
& & & & & \\
\hline 7242 & $0.001 \pm 0.001$ & 61.8 & 4.0 & 0.16 \\
BTW 1019 & $1.19 \pm 0.01$ & 0.25 & 79.2 & 0.47 \\
BTW 731 & 1.26 & \pm 0.15 & 15.7 & 2.4 & 0.67 \\
7245 & 14.7 & \pm 0.2 & 9.75 & 41.5 & 1.44 \\
7251 & 15.9 & \pm 0.2 & 13.7 & 35.9 & 1.76 \\
7243 & 20.0 & \pm 0.2 & 5.32 & 72.9 & 2.10 \\
7233 & 71.0 & \pm 0.3 & 12.7 & 61.8 & 9.46 \\
7248 & 72.0 & \pm 0.2 & 6.40 & 61.9 & 22.80 \\
7234 & - & & - & 44.0 & 860.0 \\
\hline
\end{tabular}

Table 4. Partial photochemical quantum yields of the three steps of the photochemical reaction pathway in aerated ethylene glycol.

\begin{tabular}{llll}
\hline Code name & $\varphi_{1}^{\mathrm{A}}$ & $\varphi_{2}^{\mathrm{B}}$ & $\varphi_{3}^{\mathrm{B}}$ \\
\hline Stilbene-1 & 0.016 & $0.5^{9}$ & $0.0001^{5}$ \\
BTW 1113 & 0.022 & $0.6^{7}$ & $0.0008^{7}$ \\
BTW 1115 & 0.026 & $0.3^{7}$ & $0.0004^{3}$ \\
7247 & 0.18 & $0.4^{1}$ & $0.003^{5}$ \\
BTW 1162 & 0.39 & $0.4^{7}$ & $0.002^{2}$ \\
\hline
\end{tabular}

than that for the other dyes used. This can be explained by the structure of these dyes which gives the possibility for a second isomerisation step.

All three steps of the reaction are responsible for the rate of degradation of the dyes and the ratio of the isomers in the photostationary state. Neither $Q$ nor $U$ give detailed evidence of the rate determining step and its dependence on the structure of the dye molecule. The partial photochemical quantum yields are expected to give more information. The values for the step $\mathrm{A}^{\mathrm{F}} \rightarrow \mathrm{B}$ are easily and exactly obtainable by (8). Since the used degradation yields $U$ depend to some extent on the amount of absorbed light they show a similar trend to the $\varphi_{1}^{\mathrm{A}}$ values, but these are uninfluenced by the second step. $\varphi_{2}^{\mathrm{B}}$ can be estimated according to (7). Assuming that $\mathrm{B}$ degrades relatively slowly to the consecutive photoproduct, in a first approximation its change in concentration can be described by the decrease in trans concentration caused by the relatively fast preceding equilibrium. Therefore the interesting $\varphi_{3}^{\mathrm{B}}$ values of the second linearly independent step $\mathrm{B} \rightarrow \mathrm{C}$ can be calculated from the second slope of a diagram $\ln I_{\alpha}^{\mathrm{F}}(t)$ vs. $t$ (Fig. 3) according to the rate law

$$
\dot{I}_{\alpha}^{\mathrm{F}}(t)=-I R_{3} I_{\alpha}^{\mathrm{F}}(t)
$$

with $R_{3}=\varepsilon_{\mathrm{B}}^{\prime} \varphi_{3}^{\mathrm{B}}$. For some of the dyes all the partial photochemical quantum yields are listed in Table 4. In some cases the error limits of the estimation are relatively high because of unfavourable experimental conditions (small \% cis $(s)$ ). In all those cases a calculation of $\varepsilon_{\mathrm{B}}^{\prime}$ is too inaccurate and neither $\varphi_{2}^{\mathrm{B}}$ nor $\varphi_{3}^{\mathrm{B}}$ can be determined by this simple approach.

The values of these yields depend strongly on the solvent used. In case of stilbene- $1, \varphi_{1}^{\mathrm{A}}$ decreases by one order of magnitude from methanol, water to ethylene glycol. The small values of the last solvent can also be obtained in tenside-solutions (water and $1 \%$ nonylphenyl polyethylene oxides with different chain lengths), whereby water is a favourable solvent under laser conditions [11]. The rate of disappearance is affected by the chosen wavelength of irradiation, in contrast to the quantum yields. All three steps influence the photochemical properties of the different stilbene- 1 derivatives. They have to be known for a deliberate choice of the best dye for an application as a laser dye.

A corresponding influence on the photochemical parameters has been published for the simple 
derivatives of stilbene $[18,19]$. Even though only 20 dyes have been examined, a correlation between photochemical properties and dye structure could be found by the photokinetic analysis. The partial chemical quantum yield $\varphi_{1}^{\mathrm{A}}$ (as well as the rate $U$ )

a) decreases, if in the stilbene- 1 frame

- another $-\mathrm{CH}=\mathrm{CH}$-phenyl group is inserted between the $-\mathrm{CH}=\mathrm{CH}$-double bond and the right hand diphenyl group (BTW731, BTW7242, BTW 741),

- a Cl-group is substituted in the para-position on the phenyl ring (BTW 1113, BTW 1115, BTW 7252),

- the phenyl ring is replaced by a chrysen group (BTW 1150, but this compound fluoresces at longer wavelength),

b) increases, if

- an $\mathrm{NH}_{2}$-group is substituted $(7248,7245)$, where the alkylated one is more stable (7245),

[1] B. H. Winters, H. I. Mandelberg, and W. B. Mohr, Appl. Phys. Lett. 25, (12) 723 (1974).

[2] F. P. Schäfer, Topics in Applied Physics I (Dye Lasers), Springer-Verlag, Berlin, Heidelberg, New york 1973.

[3] F. P. Schäfer, Zs. Bor, W. Lüttke, and B. Liphardt, Chem. Phys. Lett. 56, 455 (1978).

[4] V. S. Antonov and K. L. Hohla, Appl. Phys. B 32, 9 (1983).

[5] H. Mauser, Formale Kinetik, Bertelsmann Universitätsverlag, Düsseldorf 1974

[6] G. Gauglitz, T. Klink, and W. Schmid, Xth IUPAC Symposium on Photochemistry, Interlaken, Switzerland 1984.

[7] E. Fischer, J. Phys. Chem. 71, 3704 (1967).

[8] G. M. Wyman, Mol. Photochem. 6, 81 (1974).

[9] H. Jungmann, H. Güsten, and D. Schulte-Frohlinde, Chem. Ber. 101, 2690 (1968).
- a CN-group is substituted (BTW 919, 7245), whereby the stabilization effect of the $-\mathrm{CH}=\mathrm{CH}-$ phenyl group seems to be reduced,

- the phenyl ring is replaced by an indol group (7273, 7234).

The described microprocessor controlled set-up offered the possibility to measure the changes in fluorescence intensities during irradiation very exactly. Therefore a calculation of the photokinetic parameters was possible by the combined evaluation method even in complex photochemical reactions without knowledge of the spectroscopic properties of the consecutive photoproducts and their identity.

\section{Acknowledgement}

We thank the BMFT (grant $13 \mathrm{~N} 5290$ 2) and the Fond der Chemischen Industrie for personal and financial support. One of us (W. S.) was supported by the G. Rösch-Stiftung.

[10] G. Gauglitz, Z. Phys. Chem. N. F. 113, 217 (1978)

[11] E. Friz, G. Gauglitz, R. Goes, T. Klink, and W. Stooß, Xth IUPAC Symposium on Photochemistry, Interlaken, Switzerland 1984.

[12] G. Gauglitz and R. Goes, submitted for publication.

[13] C. A. Parker and C. G. Hatchard, J. Phys. Chem. 63, 22 (1953). - Trans. Faraday Soc. 50, 1213 (1954).

[14] G. Gauglitz and S. Hubig, J. Photochem. in print.

[15] E. Friz, G. Gauglitz, T. Klink, and A Lorch, Computer Enhanced Spectrosc. 1, 49 (1983).

[16] R. Goes, PhD-Thesis, University of Tübingen 1985.

[17] G. Gauglitz, J. Photochem. 5, 41 (1976).

[18] H. Görner and D. Schulte-Frohlinde, J. Amer. Chem. Soc. 101, 4388 (1979).

[19] H. Görner and D. Schulte-Frohlinde, J. Phys. Chem. 82, 2653 (1978). 